\title{
MicroRNA-30d inhibits the migration and invasion of human esophageal squamous cell carcinoma cells via the post-transcriptional regulation of enhancer of zeste homolog 2
}

\author{
RUI XIE, SHANG-NONG WU, CHENG-CHENG GAO, XIAO-ZHONG YANG, HONG-GANG WANG, \\ JIA-LING ZHANG, WEI YAN and TIAN-HENG MA \\ Department of Gastroenterology, Huai'an First People's Hospital, Nanjing Medical University, \\ Huai'an, Jiangsu 223300, P.R. China
}

Received July 19, 2016; Accepted September 6, 2016

DOI: 10.3892/or.2017.5405

\begin{abstract}
The present study was carried out to investigate the expression pattern, clinical significance and biological functions of microRNA-30d (miR-30d) in esophageal carcinogenesis. Quantitative real-time PCR was performed to detect the expression levels of miR-30d in esophageal squamous cell carcinoma (ESCC) tissues and cell lines. Then, associations between miR-30d expression and various clinicopathological features of patients with ESCC were statistically evaluated. In addition, the effects of miR-30d on the migration and invasion of two human ESCC cell lines transfected with miRNA or co-transfected with miRNA mimics and the expression vector of its target gene were determined. The results revealed that the expression levels of miR-30d were markedly decreased in ESCC tissues and cell lines, comparing with the corresponding normal controls. Notably, reduced expression of miR-30d occurred more frequently in ESCC patients with positive lymph node metastasis, moderate-poor differentiation and advanced tumor-node-metastasis stage than those with negative features. Functionally, enforced expression of miR-30d was found to inhibit cell invasion and migration of the ESCC cell lines. Luciferase reporter assay identified enhancer of zeste homolog 2 (EZH2) as a direct target gene of miR-30d. The expression level of EZH2 mRNA was negatively correlated with the expression of miR-30d in the ESCC tissues. Moreover, the inhibitory effect of miR-30d on ESCC cell motility was reversed by EZH 2 overexpression. Collectively, these findings provide convincing evidence that decreased expression of miR-30d may be implicated in esophageal carcinogenesis and progression. We also confirmed miR-30d as a
\end{abstract}

Correspondence to: Professor Tian-Heng Ma, Department of Gastroenterology, Huai'an First People's Hospital, Nanjing Medical University, Huai'an, Jiangsu 223300, P.R. China

E-mail: tianhengma258@126.com

Key words: esophageal squamous cell carcinoma, microRNA-30d, enhancer of zeste homolog 2, cell motility, carcinogenesis tumor-suppressor which may inhibit cancer cell motility by targeting EZH2, a potential therapeutic target for ESCC.

\section{Introduction}

Esophageal squamous cell carcinoma (ESCC) and esophageal adenocarcinoma represent two main histopathological subtypes of esophageal cancer, which is the eighth most common cancer and the sixth leading cause of cancer-related mortality worldwide, affecting men more than women (1). ESCC is characterized by its poor prognosis, with a 5-year survival rate less than $15 \%$ (2). Particularly, in Western countries, the incidence and the mortality rates of ESCC have steadily increased during the past few decades (3). Despite advancement in radical esophagectomy and systemic chemoradiotherapy, the clinical outcome of ESCC patients is still very poor due to the high prevalence of cell proliferation and metastasis (4). Moreover, most ESCC patients are often diagnosed at an advanced stage due to the absence of apparent symptoms and lack of early detection methods (5). Therefore, it is of great clinical significance to discover and identify tumor-specific molecular biomarkers for the early detection and effective treatment, and subsequently for the better understanding of the underlying biological mechanisms of ESCC.

MicroRNAs (miRNAs), a conserved group of endogenous, non-coding and single-stranded small RNAs 18-29 nucleotides in length, can negatively regulate gene expression by binding to the 3'-UTR of target messenger RNAs (mRNAs) post-transcriptionally, leading to translation repression or promotion of RNA degradation (6). miRNAs play crucial roles in the regulation of a variety of biological and pathological processes, including developmental timing, cellular growth, proliferation, differentiation, death, apoptosis and carcinogenesis $(7,8)$. Abnormal expression of miRNAs has been observed in multiple types of human cancers, implying that miRNAs may function as either oncogenes or tumor suppressors by the sequence-specific regulation of the corresponding target gene expression (9). miR-30d, a member of the miR-30 family, is localized in human chromosome 8q24.22 (10). Accumulating evidence has revealed that the aberrant expression of miR-30d contributes to cancer development and progression. For 
example, miR-30d downregulation was found to contribute to the development of anaplastic thyroid carcinoma through the regulation of the polycomb protein enhancer of zeste homolog 2 (EZH2) (11). miR-30d expression was found to be increased in hepatocellular carcinoma tissues and its overexpression promoted tumor invasion and metastasis by targeting Galphai2 (12). miR-30d suppressed prostate cancer cell proliferation partially by targeting Bmi-1 (13). miR-30d was found to inhibit renal carcinoma cell proliferation via the regulation of cyclin E2 expression at the post-transcriptional level (14). However, the role of miR-30d in human ESCC has not been documented. Therefore, the present study aimed to investigate the expression pattern, clinical significance and biological functions of miR-30d in esophageal carcinogenesis.

In the present study, quantitative real-time PCR was performed to detect the expression levels of miR-30d in both ESCC tissues and cell lines. Then, the associations between miR-30d expression and various clinicopathological features of patients with ESCC were statistically evaluated. In addition, the target genes of miR-30d were predicted by bioinformatic miRNA target prediction tool, and validated by western blot analysis and luciferase reporter assay. Moreover, the functions of miR-30d in migration and invasion were determined using two human ESCC cell lines following transfection with miRNA mimics or co-transfected with miRNA mimics and the expression vector of its target gene.

\section{Materials and methods}

Statement of ethics. The present study was approved by the Ethics Committee of Huai'an First People's Hospital. Prior informed consent was signed by all the patients enrolled in the present study according to guidelines of Huai'an First People's Hospital. All tissue specimens were handled and made anonymous based on ethical and legal standards.

Ethical approval. All procedures performed in the present study involving human participants were in accordance with the ethical standards of the institutional and/or national research committee and with the 1964 Helsinki Declaration and its later amendments or comparable ethical standards.

Patients and tissue samples. A total of 60 pairs of matched primary ESCC and adjacent non-cancerous esophageal mucosal tissue specimens were obtained from 60 patients who underwent esophagectomy at the Department of Gastroenterology, Huai'an First People's Hospital between Janurary 2012 and December 2015. None of the patients received radiotherapy or chemotherapy before surgery. The diagnosis was confirmed by clinical examination and histopathological analysis of the tissue specimens. The clinicopathological characteristics of all 60 ESCC patients, including age, gender, tumor location, lymph node metastasis, tumor-node-metastasis (TNM) stage (based on the 7th edition of the AJCC/UICC TNM staging system) and pathological grade are summarized in Table I. All of the samples were collected and immediately snap frozen in liquid nitrogen and stored at $-80^{\circ} \mathrm{C}$ until further use.

Cell lines and culture. A human normal esophageal cell line (HEEC) and two human ESCC cell lines (ECA109 and
KYSE410) were purchased from the Shanghai Institute of Cell Biology, Chinese Academy of Sciences (Shanghai, China). All cell lines were cultured in Dulbecco's modified Eagle's medium (DMEM; Gibco, Carlsbad, CA, USA) supplemented with $10 \%$ fetal bovine serum (FBS). Cells were cultured at $37^{\circ} \mathrm{C}$ with $5 \% \mathrm{CO}_{2}$ for further use.

Cell transfection. The transfection of human ESCC cells with miR-30d/control mimic (miR-30d/NC_mimics) and the pcDNA3.1-EZH2 expression vector (en-EZH2) (both from GenePharma Biotech, Shanghai, China) was carried out using Lipofectamine 2000 (Invitrogen, Carlsbad, CA, USA) according to the manufacturer's instructions. At $48 \mathrm{~h}$ following transfection, the ESCC cells were harvested for western blot or real-time quantitative PCR analyses.

Western blot analysis. Proteins in fresh clinical tissue specimens and cells were extracted using cell lysis buffer $(50 \mathrm{mM}$ Tris-HCl, pH 8.0, 2 mM EDTA, $1 \mathrm{mM}$ DTT, $10 \mathrm{mM} \mathrm{NaCl}$, $5 \mathrm{mg} / \mathrm{ml}$ leupeptin, $1 \% \mathrm{NP}-40,2 \mathrm{mg} / \mathrm{ml}$ pepstatin, $2 \mathrm{mg} / \mathrm{ml}$ aprotinin, $0.1 \%$ SDS and $1 \mathrm{mM}$ phenylmethylsulfonyl fluoride). Equal amounts of protein $(50 \mu \mathrm{g})$ were separated by $10 \%$ SDS-PAGE, and then transferred onto polyvinylidene difluoride membranes (Qiagen China Co., Ltd., Shanghai, China). Then, the membranes were incubated with the primary antibodies: anti-EZH2 (dilution 1:200; Zymed Laboratories Inc., South San Francisco, CA, USA) and anti-GAPDH (dilution 1:500; Abcam Inc., Cambridge, MA, USA), after blocking with $8 \%$ milk in phosphate-buffered saline (PBS; $\mathrm{pH} 7.5$ ). After that, the membranes were incubated with the appropriate horseradish peroxidase-conjugated secondary antibodies (dilution 1:1,000; Abcam Inc.) after incubation at $4^{\circ} \mathrm{C}$ overnight. Finally, the proteins were visualized using enhanced chemiluminescence reagent (Santa Cruz Biotechnology, Santa Cruz, CA, USA). The expression level of EZH2 protein was normalized to that of GAPDH protein. Each sample was examined in triplicate.

$R N A$ extraction and real-time quantitative $R T-P C R$. Total RNA in fresh clinical tissue specimens and cells was extracted using the RNeasy RNA Mini kit (Qiagen, Hilden, Germany) according to the manufacturer's instructions. First-strand cDNA was synthesized using PowerScript reverse transcriptase (Clontech, San Jose, CA, USA) according to the manufacturer's instructions. Following cDNA synthesis, real-time PCR was performed using a Fast Start Master SYBR-Green kit on a LightCycler (both from Roche Molecular Systems, Indianapolis, IN, USA) according to the manufacturer's instructions. The sequence-specific primer pairs used for quantitative PCR are listed as following: miR-30d forward, 5'-UGU AAA CAU CCC CGA CUG GAA G-3' and reverse, 5'-TGT AAA CAT CCC CGA CTG GAA GA-3'; U6 forward, 5'-CTC GCT TCG GCA GCA CA-3' and reverse, 5'-AAC GCT TCA CGA ATT TGC GT-3'; EZH2 forward, 5'-TTA CTT GTG GAG CCG CTG AC-3' and reverse, 5'-TCA GAT GGT GCC AGC AAT AG-3'; GAPDH forward, 5'-GCT GAG TAT GTC GTG GAG TC-3' and reverse, 5'-AGT TGG TGG TGC AGG ATG C-3'. Relative expression levels of miRNA and mRNA expression in fresh tissues and cells were determined using the $2^{-\Delta \Delta C t}$ method. Each sample was examined in triplicate. 
Table I. Associations between miR-30d expression and various clinicopathological characteristics of the 60 patients with ESCC.

\begin{tabular}{|c|c|c|c|c|}
\hline Clinical variables & $\begin{array}{c}\text { No. of patients } \\
(\%)\end{array}$ & $\begin{array}{c}\text { Low miR-30d } \\
\text { expression }(n, \%)\end{array}$ & $\begin{array}{l}\text { High miR-30d } \\
\text { expression }(n, \%)\end{array}$ & P-value \\
\hline \multicolumn{5}{|l|}{ Age (years) } \\
\hline$\leq 60$ & $25(41.67)$ & $13(52.00)$ & $12(48.00)$ & \multirow[t]{2}{*}{ NS } \\
\hline$>60$ & $35(58.33)$ & $19(54.29)$ & $16(45.71)$ & \\
\hline \multicolumn{5}{|l|}{ Gender } \\
\hline Male & $40(66.67)$ & $22(55.00)$ & $18(45.00)$ & \multirow[t]{2}{*}{ NS } \\
\hline Female & $20(33.33)$ & $10(50.00)$ & $10(50.00)$ & \\
\hline \multicolumn{5}{|l|}{ Tumor location } \\
\hline Upper $1 / 3$-middle1/3 & $41(68.33)$ & $23(56.10)$ & $18(43.90)$ & \multirow[t]{2}{*}{ NS } \\
\hline Lower $1 / 3$ & $19(31.67)$ & $9(47.37)$ & $10(52.63)$ & \\
\hline \multicolumn{5}{|l|}{ Lymph node metastasis } \\
\hline Negative & $24(40.00)$ & $6(25.00)$ & $18(75.00)$ & \multirow[t]{2}{*}{0.006} \\
\hline Positive & $36(60.00)$ & $26(72.22)$ & $10(27.78)$ & \\
\hline \multicolumn{5}{|l|}{ TNM stage } \\
\hline Early & $20(33.33)$ & $2(10.00)$ & $18(90.00)$ & \multirow[t]{2}{*}{$<0.001$} \\
\hline Advanced & $40(66.67)$ & $30(75.00)$ & $10(25.00)$ & \\
\hline \multicolumn{5}{|c|}{ Histological differentiation } \\
\hline Well & $18(33.33)$ & $6(33.33)$ & $12(66.67)$ & \multirow[t]{2}{*}{0.02} \\
\hline Moderate/poor & $42(66.67)$ & $26(61.90)$ & $16(38.10)$ & \\
\hline
\end{tabular}

ESCCs, esophageal squamous cell carcinomas; TNM, tumor-node-metastasis; NS, differences without statistical significance.

Cell invasion and migration assays. Cell migration and invasion abilities of human ESCC cell lines following transfection with miR-30d/NC-mimics or the co-transfection of miR-30d/en-EZH2 were evaluated using a Millicell Transwell chamber (Millipore, Billerica, MA, USA), with or without Matrigel (BD Biosciences, Franklin Lakes, NJ, USA). For the invasion and migration assays, $48 \mathrm{~h}$ following the transfection, Transwell chambers were placed into 24-well plates which were respectively precoated with or without a $5 \mathrm{ml}$ mixture of BD Matrigel and DMEM (1:1, v/v). Following incubation at $37^{\circ} \mathrm{C}$ in a humidified incubator with $5 \% \mathrm{CO}_{2}$ for $40 \mathrm{~min}$, for cell migration and invasion assays, $1 \times 10^{5}$ tumor cells in $0.1 \mu 1$ of media without FBS were plated in the upper chamber. In the lower chamber, $0.6 \mu \mathrm{l}$ of medium with $10 \%$ FBS was added. Forty-eight hours after incubation, cells on the upper surface of the Millicell chambers, non-invasive or non-migrated cells, were scraped off using a cotton swab. Tumor cells on the bottom surface of the membrane were fixed with $4 \%$ paraformaldehyde at room temperature for $30 \mathrm{~min}$ and stained with $0.1 \%$ crystal violet for $15 \mathrm{~min}$. The numbers of migrated or invasive cells were counted in five randomly selected fields under an inverted microscope (Olympus CKX41; Olympus, Tokyo, Japan). Each sample was examined in triplicate.

miRNA target prediction. Validated targets for miR-30d-5p were collected from miRTarBase (release 6.1; http://mirtarbase. mbc.nctu.edu.tw/), an experimentally validated miRNA-target interaction (MTI) database, which has accumulated more than 300 and 60,000 MTIs validated experimentally by reporter assay, western blotting, qPCR, microarray and next-generation sequencing experiments (15). While containing the largest numbers of validated MTIs, the miRTarBase provides the most updated collection by comparing with other similar, previously developed databases. In the present study, we only collected the MTIs which were validated experimentally by reporter assay, western blotting and qPCR.

Luciferase reporter assay. To determine the binding efficiency of miR-30d on the 3'UTR of EZH2 mRNA, two human ESCC cell lines were co-transfected with $\mathrm{miR}-30 \mathrm{~d} / \mathrm{NC}$-mimics and pGL3-EZH2-3'-UTR-WT/MUT. To construct the EZH2 3'-UTR luciferase reporter (pGL3-EZH2-3'-UTR), the 263-bp 3'-UTR of EZH2 mRNA was amplifed by PCR (forward, 5'-GTA TGT CGG CAT CGA AAG AG-3' and reverse, 5'-CCT GAA AGC AGT TAT TGA CA-3'). The amplified fragment was cloned into pGL3-control firefly luciferase reporter vector (Promega, Madison, WI, USA). Deletions of the seed region in the EZH2 3'-UTR constructs were introduced by site-directed mutagenesis (Eurofins MWG Operon, Ebersberg, Germany). At $48 \mathrm{~h}$ after transfection, the cells were collected and detected with the Dual-Luciferase reporter assay system (Promega, San Luis Obispo, CA, USA). Luciferase activity was measured using a Lumat LB 9507 apparatus (Berthold Technologies, Bad Wildbad, Germany). Each sample was examined in triplicate. The results are shown as relative luciferase activity, which was normalized to $\beta$-galactosidase activity.

Statistical analysis. SPSS software (version 11.0; SPSS, Inc., Chicago, IL, USA) was used to perform all statistical analyses. Data are shown as mean \pm standard deviation (SD). 
A

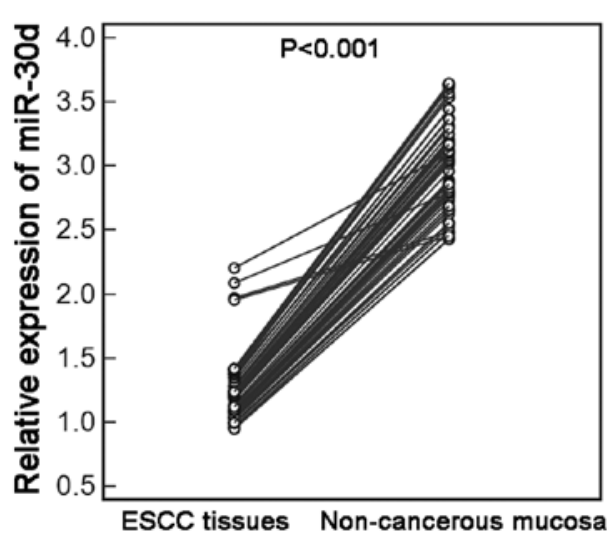

B

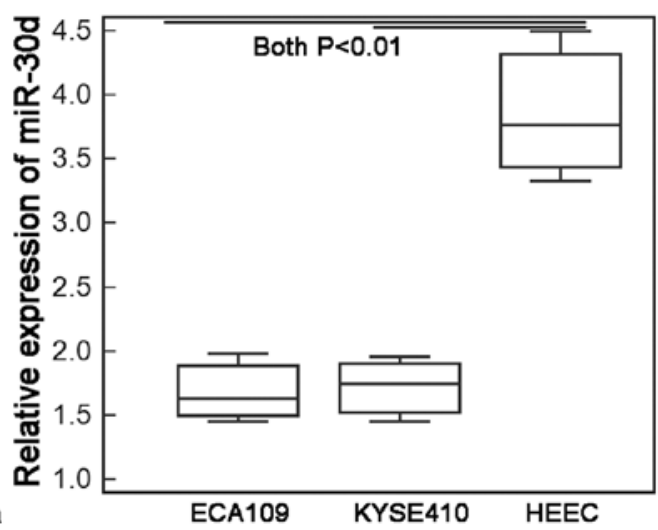

Figure 1. miR-30d expression is downregulated in ESCC tissues and cells. (A) Compared with adjacent non-cancerous esophageal mucosal tissues, respectively, the expression level of miR-30d in ESCC tissues was markedly decreased (ESCC vs. non-cancerous tissues, $1.82 \pm 0.68$ vs. $3.16 \pm 0.92$; P<0.01). (B) Compared with the human normal esophageal cell line (HEEC), respectively, the expression levels of miR-30d in two human ESCC cell lines ECA109 and KYSE410 were both markedly decreased (HEEC vs. ECA109 cells, $1.18 \pm 0.36$ vs. $2.58 \pm 0.62 ; \mathrm{P}<0.01 ;$ HEEC vs. ECA109 cells, $1.39 \pm 0.61$ vs. $2.88 \pm 0.98 ; \mathrm{P}<0.01$ ).

The differences between groups were analyzed using the Student's t-test when there were only two groups, or assessed by one-way analysis of variance (ANOVA) when more than two groups were analyzed. The correlation between miR-30d and EZH2 mRNA expression in ESCC tissues was determined by Spearman correlation analysis. The associations between miR-30d expression and various clinicopathological characteristics of the patients with ESCC were assessed using the $\chi^{2}$ test for categorical variables. A P-value $<0.05$ was considered to indicate a statistically significant result.

\section{Results}

miR-30d expression is downregulated in ESCC tissues and cells. Compared with the adjacent non-cancerous esophageal mucosal tissues and human normal esophageal cell line (HEEC), respectively, the expression levels of miR-30d in ESCC tissues, and two human ESCC cell lines ECA109 and KYSE410 were all markedly decreased [ESCC vs. non-cancerous tissues: $1.23 \pm 0.26$ vs. $3.02 \pm 0.38 ; \mathrm{P}<0.001$ (Fig. 1A); ECA109 vs. HEEC cells: $1.69 \pm 0.27$ vs. $3.87 \pm 0.59$; $\mathrm{P}<0.01$; KYSE410 vs. HEEC cells: $1.72 \pm 0.26$ vs. $3.87 \pm 0.59$; $\mathrm{P}<0.01$ (Fig. 1B)].

miR-30d downregulation is associated with aggressive tumor progression in the ESCC cases. The median value of miR-30d (1.21) expression levels in 60 ESCC tissues was used as a cut-off point to divide all 60 patients with ESCC into low miR-30d expression $(n=32)$ and high miR-30d expression $(n=28)$ groups. Table I summarizes the associations between miR-30d expression and various clinical characteristics of the patients with ESCC. Reduced expression of miR-30d occurred more frequently in ESCC patients with positive lymph node metastasis $(\mathrm{P}=0.006$; Table $\mathrm{I})$, moderate-poor differentiation $(\mathrm{P}=0.02$; Table $\mathrm{I})$ and advanced TNM stage $(\mathrm{P}<0.001$; Table I $)$ than those with negative lymph node metastasis, well differentiation and early TNM stage. There were no significant associations between miR-30d expression and patient age, gender or tumor location (all $\mathrm{P}>0.05$; Table I).
Enforced expression of miR-30d inhibits cell migration and invasion of human ESCC cells. To determine the role of miR-30d in ESCC cell motility, the migration and invasion abilities of two human ESCC cell lines ECA109 and KYSE410 transfected with the miR-30d mimics or negative control mimics were assessed. Quantitative real-time PCR showed that the expression levels of miR-30d in the ECA109 and KYSE410 cells transfected with the miR-30d mimics were markedly higher than levels in the cells tranfected with the negative control mimics (both $\mathrm{P}<0.01$; Fig. $2 \mathrm{~A}$ ). As shown in Fig. 2B, the numbers of cells that migrated through the micropore membrane were significantly decreased in the cells transfected with the miR-30d mimics compared to the numbers of cells transfected with the negative control mimics (for both ECA109 and KYSE410 cell lines; both P<0.01). Similarly, the results of the Matrigel-coated Transwell assay revealed that the numbers of miR-30d-overexpressing ESCC cells that invaded through the Matrigel were markedly lower than those in the control groups (for both ECA109 and KYSE410 cell lines; both $\mathrm{P}<0.01$; Fig. $2 \mathrm{C}$ ).

miR-30d reduces EZH2 expression by targeting the 3'-UTR of EZH2 $m R N A$. The above findings indicated that miR-30d downregulation was significantly associated with aggressive progression of patients with ESCC and enforced expression of this miRNA efficiently suppressed the motility of the ESCC cells. Next, we aimed to identify the downstream target genes which are negatively regulated by miR-30d in ESCC cells. As a result, a total of 17 candidate target genes (including ATG12, ATG2B, ATG5, BCL9, BECN1, BNIP3L, CASP3, EZH2, GNAI2, GPR78, KPNB1, NOTCH1, RUNX2, SMAD1, SNAI1, SOCS1 and TP53) for which the interaction between miR-30d was validated experimentally by reporter assay, western blotting and qPCR, were collected from miRTarBase for miR-30d. Among these genes, a previous study of Esposito et al (10) found that miR-30d directly targeted the 3'-UTR of EZH2 in anaplastic thyroid carcinoma. Growing evidence also showed that EZH2 overexpression was associated with tumor aggressiveness and poor prognosis in patients with ESCC (16-18). 
A
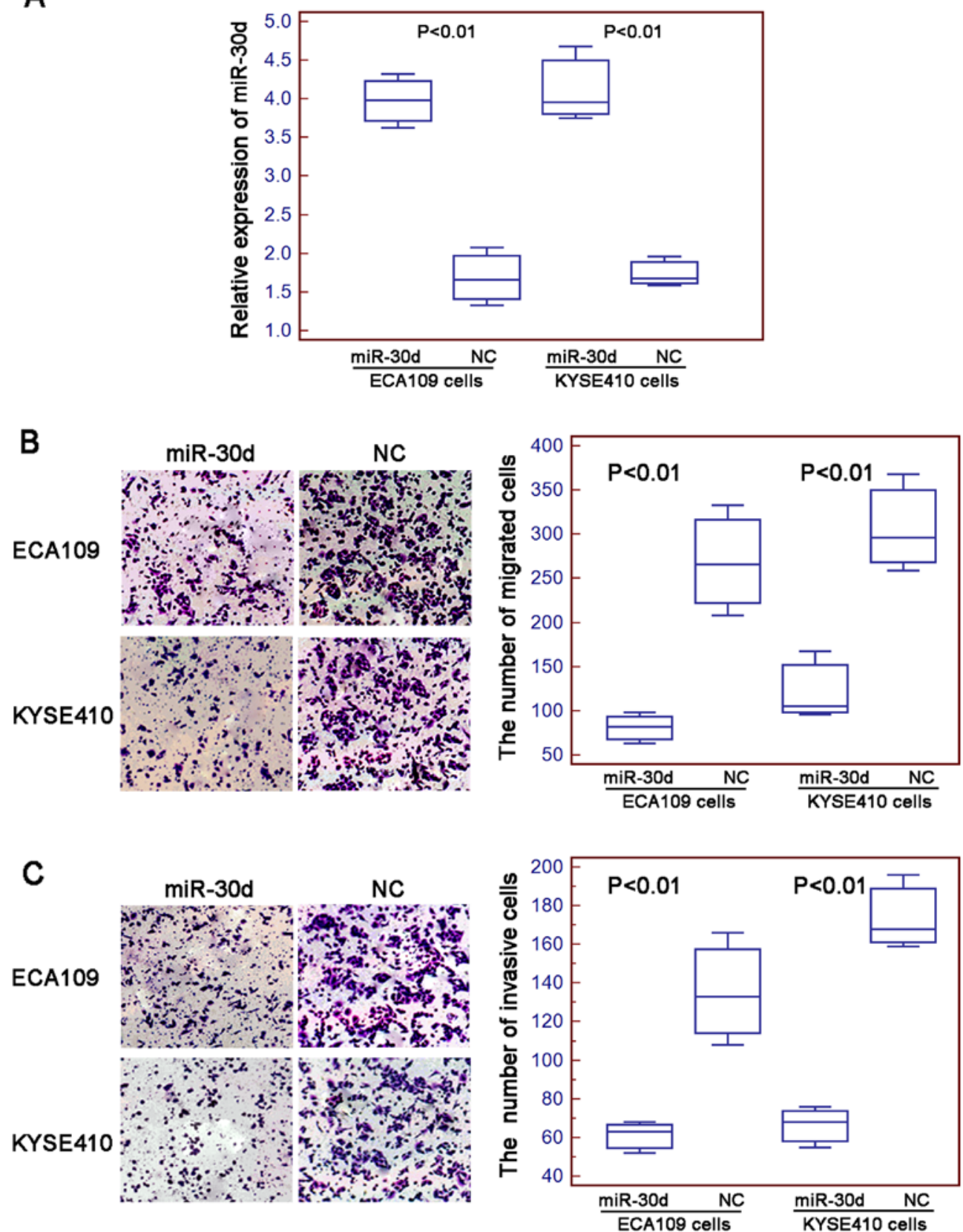

Figure 2. Enforced expression of miR-30d inhibits cell migration and invasion of human ESCC cells. (A) Quantitative real-time PCR showed that the expression levels of miR-30d in ECA109 and KYSE410 cells transfected with the miR-30d mimics were both markedly higher than level in the cells transfected with the negative control mimics (both $\mathrm{P}<0.01$ ). (B) The numbers of the migrated cells that travelled through the micropore membrane were significantly decreased in cells transfected with the miR-30d mimics compared to the cells transfected with the negative control mimics (for ECA109 and KYSE410 cells: both $\mathrm{P}<0.01$ ). (C) The numbers of cells that invaded through the Matrigel in the miR-30d-overexpressing ESCC cells were markedly lower than those in the control groups (for ECA109 and KYSE410 cells, both $\mathrm{P}<0.01$ ).

Based on this finding, we chose EZH2 as a potential target gene of miR-30d in ESCC cells.

The luciferase activities in ECA109 and KYSE410 cells co-transfected with miR-30d/NC-mimics and pGL3-EZH23'-UTR-WT/MUT were measured by luciferase report assay. As shown in Fig. 3A and B, ECA109 and KYSE410 cells co-transfected with miR-30d-mimics and pGL3-EZH2-3-UTRWT both displayed a significant decrease in reporter activity in comparison with the activity of cells co-transfected with the NC-mimics and the pGL3-EZH2-3'-UTR-WT plasmid.
However, there were no differences with statistical significance in the reporter activity between ECA109 and KYSE410 cells co-transfected with the miR-30d-mimics and the pGL3-EZH2-3'-UTR-MUT plasmid and cells co-transfected with the NC-mimics and the pGL3-EZH2-3'-UTR-MUT plasmid (Fig. 3A and B).

In addition, enforced expression of miR-30d markedly inhibited the endogenous expression of EZH2 mRNA and protein in the ECA109 and KYSE410 cells (Fig. 3C and D). Moreover, our Spearman's correlation analysis showed a 
A

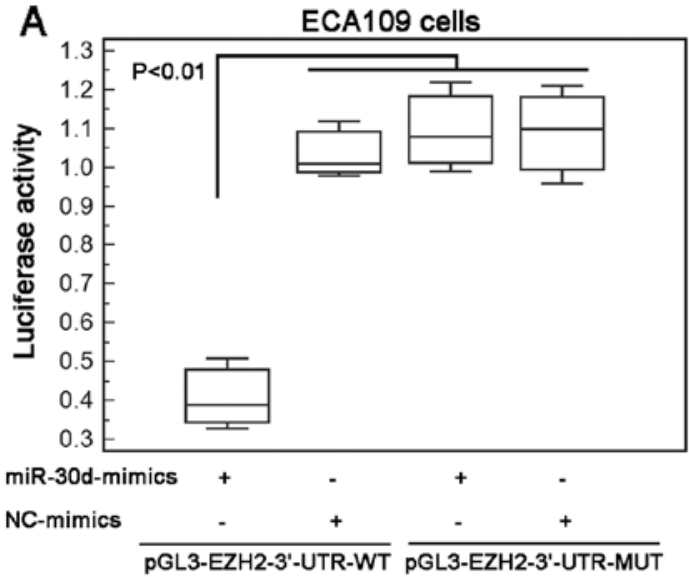

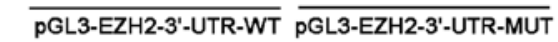

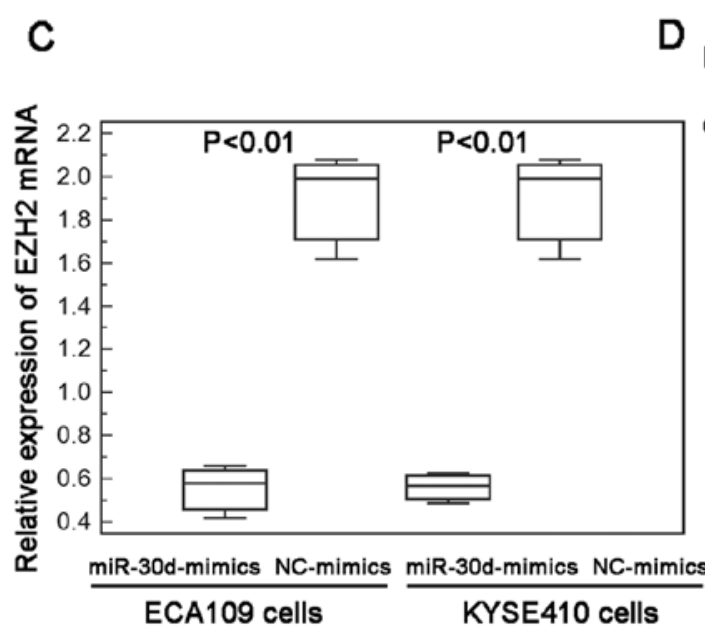

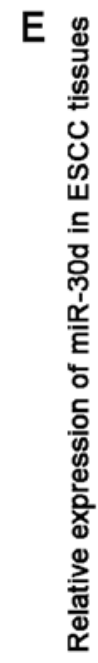

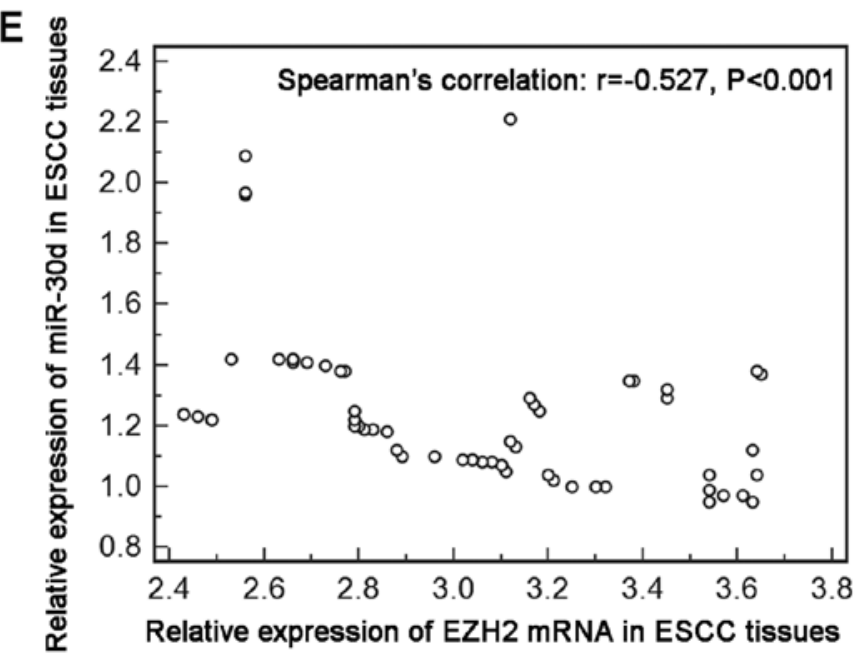

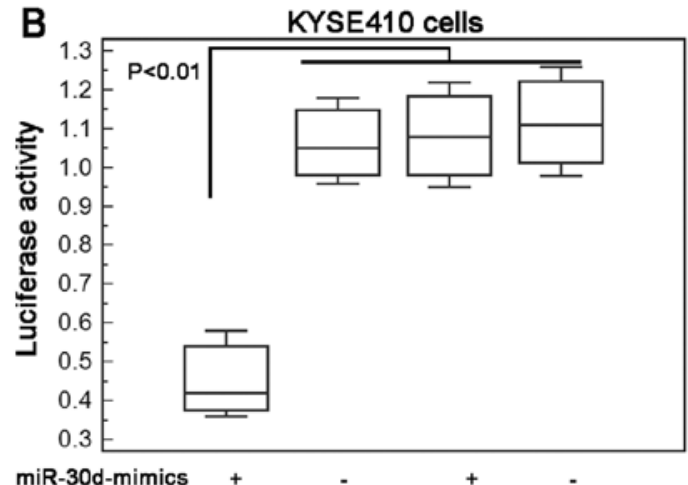

$+\quad-$

NC-mimics $\frac{-}{\text { pGL3-EZH2-3'-UTR-WT }} \frac{+}{\text { pGL3-EZH2-3'-UTR-MUT }}$

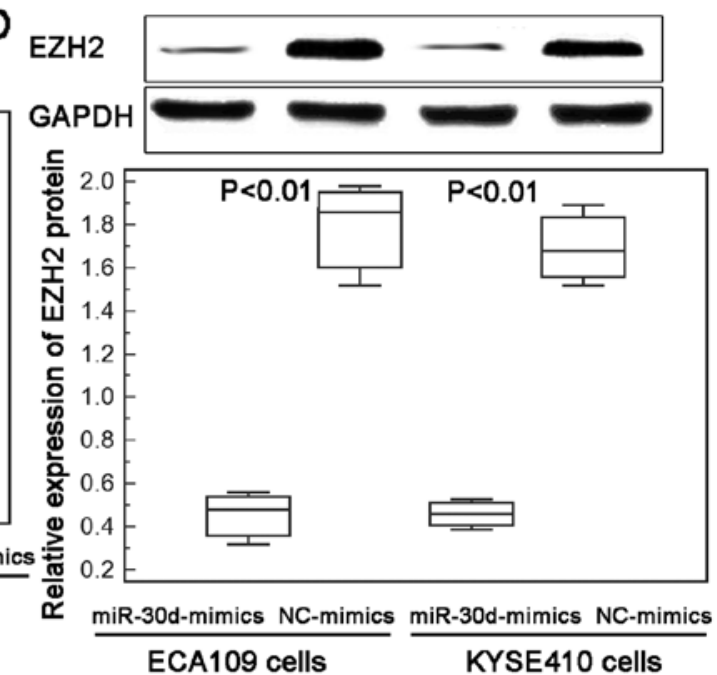

Figure 3. EZH2 is a direct target of miR-30d in human ESCC cells. (A and B) ECA109 and KYSE410 cells co-transfected with the miR-30d-mimics and pGL3EZH2-3'-UTR-WT both displayed a significant decrease in reporter activity in comparison with the activity noted in cells co-transfected with the NC-mimics and the pGL3-EZH2-3'-UTR-WT plasmid. However, there were no differences with statistical significance in the reporter activity between ECA109 and KYSE410 cells co-transfected with the miR-30d-mimics and pGL3-EZH2-3'-UTR-MUT plasmid and cells co-transfected with the NC-mimics and pGL3EZH2-3'-UTR-MUT plasmid.(C and D) Enforced expression of miR-30d markedly inhibited the endogenous expression of EZH2 mRNA and protein in the ECA109 and KYSE410 cells, respectively. (E) Spearman's correlation analysis showed a negative correlation between miR-30d and EZH2 mRNA expression in the ESCC tissues (Spearman's correlation, $\mathrm{r}=-0.527$; $\mathrm{P}<0.001$ ).

negative correlation between miR-30d and EZH2 mRNA expression in ESCC tissues (Spearman's correlation: $r=-0.527$, $\mathrm{P}<0.001$; Fig. 3E).

These findings provide evidence that EZH2 may be a direct target of miR-30d in human ESCC cells.
Suppression of migration and invasion of ESCC cells by miR-30d is reversed by the overexpression of EZH2. To determine whether miR-30d suppressed cell migration and invasion of human ESCC cells via regulating its target gene EZH2, the motility capacities of the ECA109 and KYSE410 cells 


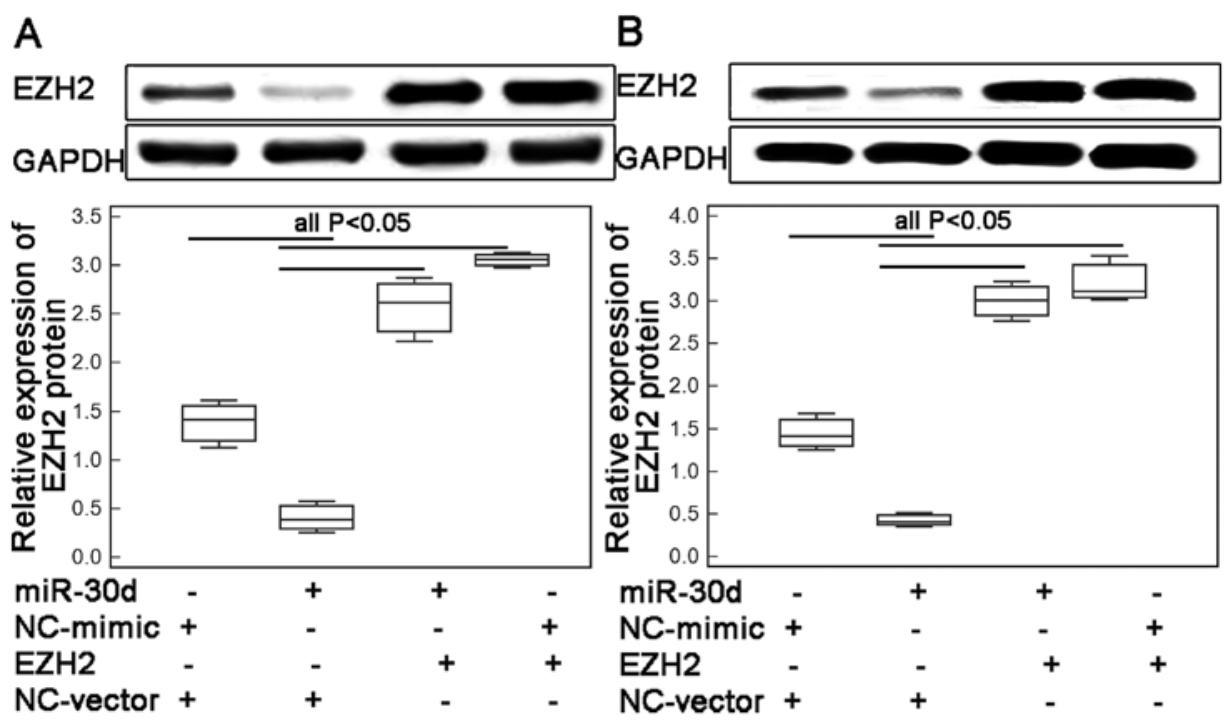

Figure 4. Relative expression levels of EZH2 protein in human ESCC cells co-transfected with the miR-30d/NC-mimcs and EZH2/NC-expression vectors. (A and B) Western blot analysis showed that endogenous expression levels of EZH2 protein in the ECA109 and KYSE410 cells transfected with the miR-30d mimic in the presence of the EZH2 expression vector were both significantly higher than these levels in the cells co-transfected with the miR-30d mimic and vector control (both $\mathrm{P}<0.01$, respectively).

co-transfected with the miR-30d-mimics and the pcDNA3.1EZH2 expression vector were evaluated. As a result, western blot analysis showed that the endogenous expression levels of EZH2 protein in the ECA109 and KYSE410 cells transfected with the miR-30d mimic in the presence of the EZH2 expression vector were both significantly higher than the levels in the cells co-transfected with the miR-30d mimic and vector control (both $\mathrm{P}<0.01$; Fig. $4 \mathrm{~A}$ and $\mathrm{B}$, respectively). Functionally, a markedly higher number of ESCC cells co-transfected with the miR-30d mimics and pcDNA-EZH2 expression vector migrated through the micropore or invaded through the Matrigel (all $\mathrm{P}<0.05$; Fig. 5), compared with the numbers of cells co-transfected with the miR-30d mimics and vector control.

\section{Discussion}

Accumulating evidence has revealed that miRNA-based molecular alterations may be implicated in the initiation and progression of human ESCC by activating or suppressing multiple malignant processes $(19,20)$. However, the underlying mechanisms of esophageal carcinogenesis have not been fully elucidated. In the present study, we demonstrated that the expression level of miR-30d in ESCC tissues was markedly lower than the level in adjacent non-cancerous esophageal mucosal tissues, which was similar with the observations observed using two human ESCC cell lines and one human normal esophageal cell line. Then, we also found that the reduced expression of miR-30d was significantly associated with the status of lymph node metastasis and the histological differentiation of ESCC tissues, as well as with the TNM stage. Morover, decreased expression of miR-30d was negatively correlated with increased expression of EZH2 in ESCC tissues. Moreover, our data showed that increased miR-30d expression efficiently inhibited cell migration and invasion by reducing the expression of EZH2. These findings imply a crucial role for the aberrant expression of miR-30d in the carcinogenesis and metastasis of ESCC cells.

The differential expression patterns of miR-30d in normal and cancerous cells suggest the roles of this miRNA in sustaining normal physiological conditions as well as its implications in cancers. It functions as either an oncogene or a tumor suppressor by regulating the expression of its target gene (11-14). Our data in the present study identified a tumor-suppressive role of miR-30d in human ESCC and also identified EZH2 as a direct target gene of this miRNA. As a member of polycomb repressive complex (PRC)2, EZH2 functions as a histone methyl transferase (21). Under a normal condition, EZH2 was found to control cell growth, cell proliferation and cell cycle (22). During carcinogenesis, EZH2 overexpression was originally observed in hematologic malignancies, and then its amplification was confirmed in various human types of cancers, such as oral cancer, ESCC, breast, lung and gastric cancer, hepatocellular carcinoma, colon, prostate, bladder and endometral cancer (16-18,23-28). Aberrant expression of EZH2 was reported to be associated with tumor aggressive progression and poor prognosis in patients with many types of human cancers. Particularly, in ESCC, He et al (16) indicated that overexpression of EZH2 was found in $>50 \%$ of ESCC patients, and was closely correlated with increased cell proliferation, high histopathological grade, regional and distant lymph node metastasis and lack of clinical complete response to chemoradiotherapy, as well as adverse patient outcome of ESCC patients treated with definitive chemotherapy; Ha et al (17) also identified the co-expression of EZH2 and another member of PRC1-Bmi1 as an independent poor prognostic factor in ESCC. In the present study, EZH2 was confirmed to be a direct target of miR-30d based on a luciferase reporter system, the western blot assay and the spearman correlation analysis of the expression levels 

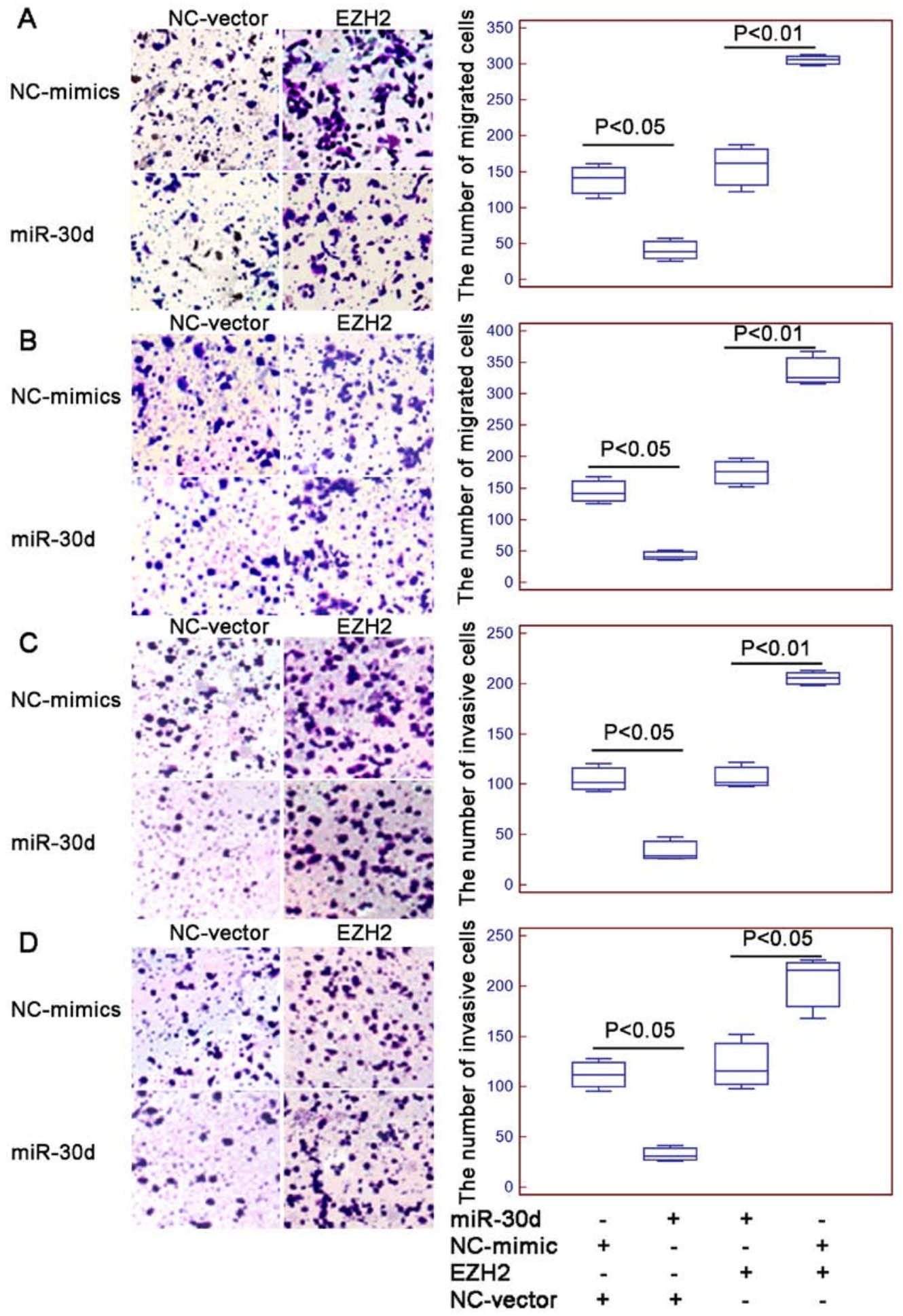

Figure 5. Suppression of migration and invasion of ESCC cells by miR-30d is reversed by the overexpression of EZH2. (A and B) Markedly higher numbers of ECA109 and KYSE410 cells, respectively, co-transfected with the miR-30d mimics and pcDNA-EZH2 expression vector migrated through the micropore (all P<0.05). (C and D) Markedly higher numbers of ECA109 and KYSE410 cells, respectively, co-transfected with the miR-30d mimics and pcDNA-EZH2 expression vector invaded through the Matrigel (all $\mathrm{P}<0.05$ ).

of miR-30d and EZH2 mRNA in ESCC tissues. Rescue experiments also demonstrated that the inhibitory effects of miR-30d in cell migration and invasion of ESCC cells were reversed by the enforced expression of EZH2, which indicate a role of the miR-30d-EZH2 axis in esophageal malignant progression.
In conclusion, these findings provide convincing evidence that the decreased expression of miR-30d may be implicated in esophageal carcinogenesis and progression. We also confirmed miR-30d as a tumor suppressor which may inhibit cancer cell motility by targeting EZH2, implying a potential therapeutic target for ESCC. 


\section{References}

1. Jemal A, Bray F, Center mM, Ferlay J, Ward E and Forman D Global cancer statistics. CA Cancer J Clin 61: 69-90, 2011.

2. Enzinger PC and Mayer RJ: Esophageal cancer. N Engl J Med 349: 2241-2252, 2003.

3. Tan C, Qian X, Guan Z, Yang B, Ge Y, Wang F and Cai J: Potential biomarkers for esophageal cancer. Springerplus 5: 467, 2016.

4. Ohashi S, Miyamoto S, Kikuchi O, Goto T, Amanuma Y and Muto M: Recent advances from basic and clinical studies of esophageal squamous cell carcinoma. Gastroenterology 149: $1700-1715,2015$.

5. Kang X,Chen K,Li Y,Li J,D'Amico TA and Chen X: Personalized targeted therapy for esophageal squamous cell carcinoma. World J Gastroenterol 21: 7648-7658, 2015.

6. Yan X, Xu H and Yan Z: Functional perspective and implications of gene expression by noncoding RNAs. Cancer Transl Med 1: 137-152, 2015.

7. Svoronos AA, Engelman DM and Slack FJ: OncomiR or tumor suppressor? The duplicity of microRNAs in cancer. Cancer Res 76: 3666-3670, 2016.

8. Otero-Albiol D and Felipe-Abrio B: MicroRNA regulating metabolic reprogramming in tumor cells: New tumor markers. Cancer Transl Med 2: 175-181, 2016

9. Rupaimoole R, Calin GA, Lopez-Berestein G and Sood AK: miRNA deregulation in cancer cells and the tumor microenvironment. Cancer Discov 6: 235-246, 2016.

10. Yang XJ, Si RH, Liang YH, Ma BQ, Jiang ZB, Wang B and Gao P: Mir-30d increases intracellular survival of Helicobacter pylori through inhibition of autophagy pathway. World J Gastroenterol 22: 3978-3991, 2016.

11. Esposito F, Tornincasa M, Pallante P, Federico A, Borbone E, Pierantoni GM and Fusco A: Down-regulation of the miR-25 and miR-30d contributes to the development of anaplastic thyroid carcinoma targeting the polycomb protein EZH2. J Clin Endocrinol Metab 97: E710-E718, 2012.

12. Yao J, Liang L, Huang S, Ding J, Tan N, Zhao Y, Yan M, Ge C, Zhang Z, Chen T, et al: MicroRNA-30d promotes tumor invasion and metastasis by targeting Galphai2 in hepatocellular carcinoma. Hepatology 51: 846-856, 2010.

13. Kobayashi N, Uemura H, Nagahama K, Okudela K, Furuya M, Ino Y, Ito Y, Hirano H, Inayama Y, Aoki I, et al: Identification of miR-30d as a novel prognostic marker of prostate cancer. Oncotarget 3: 1455-1471, 2012.

14. Yu H, Lin X, Wang F, Zhang B, Wang W, Shi H, Zou B and Zhao J: Proliferation inhibition and the underlying molecular mechanisms of microRNA-30d in renal carcinoma cells. Oncol Lett 7: 799-804, 2014
15. Chou CH, Chang NW, Shrestha S, Hsu SD, Lin YL, Lee WH, Yang CD, Hong HC, Wei TY, Tu SJ, et al: miRTarBase 2016: Updates to the experimentally validated miRNA-target interactions database. Nucleic Acids Res 44: D239-D247, 2016.

16. He LR, Liu MZ, Li BK, Jia WH, Zhang Y, Liao YJ, Chen YC, Zhang LJ, Guan XY, Zeng YX, et al: High expression of EZH2 is associated with tumor aggressiveness and poor prognosis in patients with esophageal squamous cell carcinoma treated with definitive chemoradiotherapy. Int J Cancer 127: 138-147, 2010.

17. Ha SY and Kim SH: Co-expression of Bmil and EZH2 as an independent poor prognostic factor in esophageal squamous cell carcinoma. Pathol Res Pract 208: 462-469, 2012.

18. Huang SD, Yuan Y, Zhuang CW, Li BL, Gong DJ, Wang SG, Zeng ZY and Cheng HZ: MicroRNA-98 and microRNA-214 post-transcriptionally regulate enhancer of zeste homolog 2 and inhibit migration and invasion in human esophageal squamous cell carcinoma. Mol Cancer 11: 51, 2012.

19. Sakai NS, Samia-Aly E, Barbera M and Fitzgerald RC: A review of the current understanding and clinical utility of miRNAs in esophageal cancer. Semin Cancer Biol 23: 512-521, 2013.

20. Mayne GC, Hussey DJ and Watson DI: MicroRNAs and esophageal cancer - implications for pathogenesis and therapy. Curr Pharm Des 19: 1211-1226, 2013.

21. Gall Trošelj K, Novak Kujundzic R and Ugarkovic D: Polycomb repressive complex's evolutionary conserved function: The role of EZH2 status and cellular background. Clin Epigenetics 8: 55, 2016.

22. Italiano A: Role of the EZH2 histone methyltransferase as a therapeutic target in cancer. Pharmacol Ther 165: 26-31, 2016.

23. Herviou L, Cavalli G, Cartron G, Klein B and Moreaux J: EZH2 in normal hematopoiesis and hematological malignancies. Oncotarget 7: 2284-2296, 2016.

24. Kim KH and Roberts CW: Targeting EZH2 in cancer. Nat Med 22: 128-134, 2016.

25. Martínez-Fernández M, Rubio C, Segovia C, López-Calderón FF, Dueñas M and Paramio JM: EZH2 in bladder cancer, a promising therapeutic target. Int J Mol Sci 16: 27107-27132, 2015.

26. Yin Y, Qiu S and Peng Y: Functional roles of enhancer of zeste homolog 2 in gliomas. Gene 576: 189-194, 2016.

27. Xu B, Konze KD, Jin J and Wang GG: Targeting EZH2 and PRC2 dependence as novel anticancer therapy. Exp Hematol 43: 698-712, 2015.

28. Han Li C and Chen Y: Targeting EZH2 for cancer therapy: Progress and perspective. Curr Protein Pept Sci 16: 559-570, 2015 . 